\title{
AUTOMATED MARINE OIL SPILL DETECTION USING DEEP LEARNING INSTANCE SEGMENTATION MODEL
}

\author{
${ }^{1}$ Shamsudeen Temitope Yekeen* and ${ }^{1}$ Abdul-Lateef Balogun \\ ${ }^{1}$ Geospatial Analysis and Modelling (GAM) Research Group, Department of Civil and Environmental Engineering, Universiti \\ Teknologi PETRONAS (UTP), 32610 Seri Iskandar, Perak, Malaysia. \\ (Temishams@gmail.com, Shamsudeenyekeen1@gmail.com)
}

KEYWORDS: Oil Spill, Deep Learning, Detection, Mask R-CNN, Instance Segmentation

\begin{abstract}
This study developed a novel deep learning oil spill instance segmentation model using Mask-Region-based Convolutional Neural Network (Mask R-CNN) model which is a state-of-the-art computer vision model. A total of 2882 imageries containing oil spill, look-alike, ship, and land area after conducting different pre-processing activities were acquired. These images were subsequently sub-divided into $88 \%$ training and $12 \%$ for testing, equating to 2530 and 352 images respectively. The model training was conducted using transfer learning on a pre-trained ResNet 101 with COCO data as a backbone in combination with Feature Pyramid Network (FPN) architecture for the extraction of features at 30 epochs with 0.001 learning rate. The model's performance was evaluated using precision, recall, and F1-measure which shows a higher performance than other existing models with value of $0.964,0.969$ and 0.968 respectively. As a specialized task, the study concluded that the developed deep learning instance segmentation model (Mask R-CNN) performs better than conventional machine learning models and semantic segmentation deep learning models in detection and segmentation of marine oil spill.
\end{abstract}

\section{INTRODUCTION}

The occurrence of oil spill has increased over time, due to the growth in global population resulting into intensive oil exploration and transportation (Yu et al., 2018, Yekeen et al., 2019, Balogun et al., 2020). Which has caused severe environmental, health and social-economic phenomenon (Jiao et al., 2019). Evidence to this, is the differences in major oil spill between 1970s, 1980s and 1990s with one million, 661,000, and 702,000 tons of oil spills respectively and 2000 with 74,000 tones (Chen et al., 2019). The world at large has suffered several oil spills which includes, Canada Atlantic Empress (2,100,000-2,400,000 Barrels), Angola ABT Summer (1,800,000 -1,900,000 barrels), France Amoco Cadis oil Tanker $(1,600,000-1,700,000$ Barrels), South African ship tank fire of Castillo De Bellver (1,850,000 barrels), West Indies Fergana Valley $(2,000,000$ plus Barrels), Mexico Ixtoc (3,300,000 Barrel), Persian Gulf Iran -Iraq War (1,900,000 Barrels), Italy, Uzbekistan and Kuwait first gulf war in 1991 (240,000,000 gallons) (Balogun et al., 2020). Thus, the causes of the oil spills can be categories into ship collision, grounding, hull failure, explosion/fire, and oil rig explosion (Michel and Fingas, 2015, Balogun et al., 2020). The effects of oil spill becomes more extreme due to the inability to rapidly identify the source and extent of the spill due to the predominant use of traditional methods like site visitation and camera etc which have limited area coverage.

Considering this limitation, remote sensing technologies has appear to be more promising, particularly since it can be deployed at any time (Robbe and Hengstermann, 2006,
Fingas, 2016, Ozigis et al., 2019b). The use of remote sensing technology for detecting and monitoring oil spill in marine environment have been well defined and documented in literature (Fingas and Brown, 2018), with Synthetic Aperture Radar (SAR) being the most prevalent, due to its ability to operate at any period of the day and under different weather conditions (Migliaccio et al., 2008). Despite the successes of remote sensing in this regard, a major drawback remains the similar visual appearance of oil spill and other elements such as natural films, wind front areas, wind shadow at areas close to island, ship wake, sea grass which are refers to as look-alike in the satellite imagery (Fingas and Brown, 2018, Leifer et al., 2012, Fingas and Brown, 2014). So as to overcome the false visual appearance of look-alike as oil slick, different classification models for discrimination of oil spill and look-alike have been developed over time. These models follows a three step approach of dark-spot identification, feature extraction and dark-spot classification using adaptive threshold (Vyas et al., 2015), statistics (Skøelv and Wahl, 1993), machine learning classifiers like SVM (Wan and Cheng, 2013), decision tree and ANN (Singha et al., 2012, Singha et al., 2013). Limited accuracy and non-reliability due inconsistence in feature selection, likely false identification of non-oil slick feature during dark spot identification as well the inapplicability of the model when the oil slick appears thin in the imagery are major challenge of this model. In addition to the inability to apply an end-to-end trainable framework.

Due to these shortfalls, in recent time, deep learning models are also been developed for the discrimination of oil spill and look-alike as a result of its ability to accurately and

\footnotetext{
* Corresponding Author
} 
adequately extract features in addition to its autonomous learning (Zeng and Wang, 2020, Zhao et al., 2020). However, past studies in this direction has being limited to mainly semantic segmentation model (Krestenitis et al., 2019, Gallego et al., 2019), which are not able to localize and segment accurately and simultaneously. Thus, in order to provide a model that is capable of detecting and segmenting oil spill and look-alike in addition to other features that are included within the surrounding of the oil spill like ship and land area. This study has developed an instance segmentation deep learning model using MaskRegion- based Convolutional Neural Network (Mask R$\mathrm{CNN}$ ) with the aim of achieving a high precision detection, recognition and segmentation model, that can learn from the shape and texture for localization and target detection of oil spill, lookalike, ship and land area. To the knowledge of the authors, this is perhaps the first study that implements deep learning instance segmentation model to SAR imagery for marine oil spill detection.

For flow of readership, other sections of the study are organized as follows: Section 2 entails the materials and methods used stating the type and sources of data used, the pre-processing techniques and the processed involved in the development of the model. Section 3 gives detail result and discussion of the model output, in addition to comparison of the model with other existing models. The Section 4 therefore give a conclusion remark and also states the future outlook to improving the model accuracy.

\section{MATERIALS AND METHODS}

\subsection{Data Pre-Processing}

To achieve the aim of this study, a two section methodological approach was developed. The first section entails the pre-processing that starts with the acquiring of the oil spill location, time and causes inventory for the year 2012 to 2017. It was based on this list that sentinel 1A and B imagery for this period were acquired from SAR Capernicus database with $250 \mathrm{~km}$ coverage and pixel spacing $10 \times 10$ meters. The vertical polarization transmitted-vertical polarization received (VV) from the acquired imagery with dark spot was subjected to confirmation as oil slick using the oil spill incidents list.

The areas with identified oil spill was sub-settled/cropped and rescaled to $1200 \mathrm{X} 1200$ pixels. This is in the bid to so reduce the computation and running time of the proposed model. Radiometric calibration and single layer spackle filter was also applied to de-noise to images. And a linear transformation was also done using decibel (dB) (Eq. 1) to luminous the value conversion.

$\sigma 0(\mathrm{~dB})=10 * \log 10(\operatorname{abs}(\sigma 0))$

Where $\sigma 0(\mathrm{~dB})=$ Value of image Backscattering in $\mathrm{dB}$

A total of 2882 imageries that contains oil spill, lookalike, land area and ships. Although the proportion of the elements differs since it is not all the oil spill that are close to the land area, and some oil slick were clearly standing without look- alike or ship and vise-versa. In other word it is not all the images that contain oil spill, look-alike, ship, and land area.

\subsection{Mask R-CNN Oil Spill Detection and Segmentation Model Development}

The second section constitute the training, validation and testing of the model using Mask R-CNN. The images were all labeled with the ground truth data and subsequently divided into $88 \%$ training and $12 \%$ for testing amounting to 2530 and 352 images respectively to have a substantial training and testing data.

The Mask R-CNN model involves a three stage processes as presented in Figure 1, with the first been the backbone network extraction feature map from the input imageries (training data) using ResNet 101 deep neural network model which has being trained on the COCO data by Abdulla (2017) in addition to the Feature Pyramid Network (FPN) architecture that enables extraction of feature at different scale because of the variation in size of the different elements. Second stage was the sending of the output from the feature map backbone to the Regional Proposal Network (RPN) to generate regions of interest (RoI). RoI output mapped from RPN which has extract the equivalent target features in the shared feature maps and output from Fully Convolutional and Fully Convolutional networks for classification and instance segmentation of oil spill, looalike, land area and ship. The model was trained using a learning rate of 0.001 and 30 epoch with 1 image per batch (See Table 1 for Model configuration Parameters).

\begin{tabular}{cc}
\hline Backbone & {$[4,8,16,32,64]$} \\
Batch size & 0 \\
Batch size & $0.10 .10 .20 .2]$ \\
Detection maximum instance & 100 \\
Detection minimum confidence & 0.7 \\
Detection NMS threshold & 0.3 \\
FPN Class IF FC layers size & 1024 \\
Gradient clip norm & 5.0 \\
Image channel count & 3 \\
Image maximum dimension & 1024 \\
Train_BN & False \\
Image MATA Size & 93 \\
RPN anchor ratios & $0.5,1,2]$ \\
RPN anchor scales & $128,256,512>$ \\
RPN_Train_Anchors_Per_Image & 256 \\
USE_Mini_Mask & True \\
USE_RPN_ROIS & True \\
Validation steps & 50 \\
Learning rate & 0.001 \\
Learning momentum & 0.9 \\
Training Epochs & 30 \\
RPN NMS threshold & 0.7 \\
\hline
\end{tabular}

Table 1: Mask R-CNN Parameters Configuration 


\section{RESULT AND DISCUSSION}

\subsection{Model Accuracy Evaluation}

The model was evaluated using 352 testing images that includes oil spill, lookalike, ship, and land area at different proportion. This accuracy were measured using three different performance indices that includes precision, recall and $F_{1}$ measure value all of which were derived from the confusion matrix.

$F_{1}$ accuracy is represented by (Eq.2) which connote the harmonic mean of precision and sensitivity recall that ascertain the out of bag error of the model (Ozigis et al., 2019a). In other term, this means the extent at which the predicted detection mask boundary generated of the Mask R$\mathrm{CNN}$ aligns well with the ground truth boundary.

$\mathrm{F}_{1}$ accuracy $=2 * \frac{\text { Precision } * \text { Recall }}{\text { Recall }+ \text { Precision }}$

The Precision is the division of the true positive pixels (TP) by the sum of true positive pixels and false positive (FP) pixels (Eq. 3). While the recall is the division of true positive pixels by the sum of true positive pixel and false negative (FN) pixel (Eq. 4).

$$
\text { Precision }=\frac{\mathrm{TP}}{\mathrm{TP}+\mathrm{FP}}
$$

Recall $=\frac{\mathrm{TP}}{\mathrm{TP}+\mathrm{FN}}$ with a precision, recall and $\mathrm{F}_{1}$ measure value of $(0.987$, 0.976 and 0.982$)$ for oil spill as against the $(0.927,0.966$ and 0.946 ) for lookalike. Ship measured $(0.988,0.994$ and 0.991$)$ and land area measures $(0.955,0.941$, and 0.948). Although the model accuracy measure for the ship class outperforms other classes which was as a result of the fact that ship has a more definite shape than other elements understudy. The matter of concern which are oil spill and look-alike were adequately detected and segmented with high accuracy value from the evaluation indicators as illustrated on Table 2 . Although, the lower accuracy of the look-alike in comparison to the oil spill can be largely linked to the complexity in the shape and variation in the size of the lookalikes.

\begin{tabular}{cccc}
\hline Class & Precision & Recall & F1 \\
\hline Oil Spill & 0.987 & 0.976 & 0.982 \\
\hline Look-alike & 0.927 & 0.966 & 0.946 \\
\hline Ship & 0.988 & 0.994 & 0.991 \\
\hline Land Area & 0.955 & 0.941 & 0.948 \\
\hline $\begin{array}{c}\text { Mask R-CNN Average } \\
\text { Accuracy }\end{array}$ & 0.964 & 0.969 & 0.968 \\
\hline
\end{tabular}

Table 2: Result of Model Performance Evaluation from confusion matrix

The result revealed that the model was able to adequately detect and segment oil spill, look-alike, ship and land area

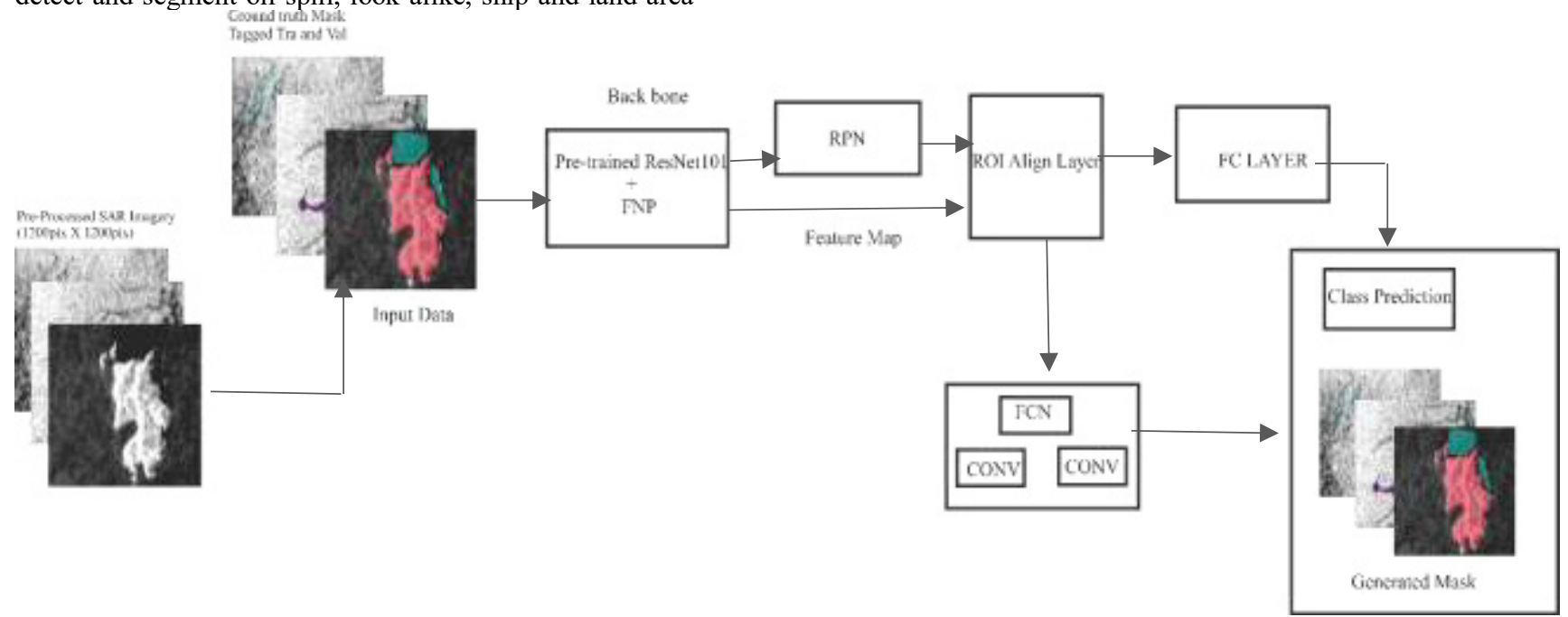

Figure 1: General Architecture of the Deep Learning Oil Spill Detection and Segmentation Model with Mask RCNN

\subsection{Model Quantitative Assessment and Comparison with Existing Methods and Models}

The visual output performance of the model are presented in Figure 2.The assessment of the model indicated that the ground truth mask of the different classes ship (yellow), look-alike (cyan), oil spill (purple) and land area (pink) under study were adequately detected and segmented by the model in the SAR imagery. Although, Ship depicts to be relatively better segmented and detected than other due to its definite shape. Never the less, oil spill was well detected and segmented by the model, taking into consideration the higher performance as represented in Table 2 compared to look- 
alike which are likely due to the more complex appearance of look-alike.

In comparison with existing models like statistical, traditional machine learning, and other semantic deep learning models (Topouzelis et al., 2007, Liu et al., 2010, Singha et al., 2013, Ma, 2016, Mera et al., 2017, Gallego et al., 2018, Krestenitis et al., 2019, Zhang et al., 2020) this study has incorporated other elements that has significant influence on the detection of oil spill, which include ship and land area, that were absent in other studies. The earlier and existing methods have been affected by the errors caused by speckle noise and the global gray unevenness in SAR imagery, leading to a reduction in the dark spot identification thereby affecting the statistical feature selection and classification of oil spill and look-alike (Gallego et al.,

A
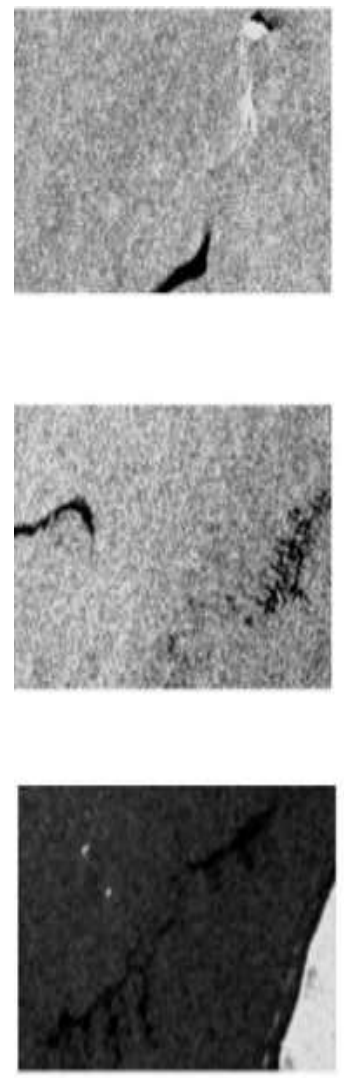

B
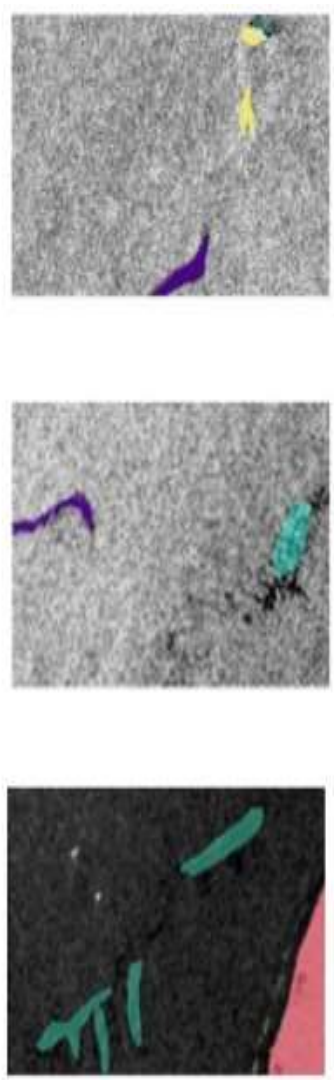

2018). As a means of eradicating these errors, sparkle filtering and calibration as well as $\mathrm{dB}$ conversion to aid global gray unevenness in the images used in the preprocessing stage of this study were employed before it was later used for the model training and validation. Also, the statistical methods and the machine learning models do not possess an end-to-end training framework that reduced their accuracy and reliability, which the Mask R CNN model developed has overcome, thereby giving a more accurate and precise detection and segmentation.

Figure 2. Examples of Qualitative Output of the Mask R-CNN model, the Ground truth and SAR Imagery

\section{CONCLUSION}

A rapid, accurate and reliable mechanism for oil spill detection is a fundamental aspect of marine oil spill pollution decision support system. Bearing in mind the complex nature of marine oil spill detection, a sophisticated model that enables learning from past scenes in term of shape and texture for rapid and accurate recognition and segmentation is required. This study has developed an instance segmentation deep learning model for oil spill detection and segmentation using Mask R-CNN in a two sectional methodological approach which is advanced and different from other exiting methods as it uses pixel value for inference. Comparison of the Mask R-CNN model with other machine learning, deep learning and statistical models indicates that the Mask R-CNN outperforms other exiting models in oil spill detection and segmentation. As a future direction, there is need for the development of a large database for oil spill SAR imagery data this is with the view to further improve the accuracy of the model and enable development of oil spill deep learning framework. 


\section{ACKNOWLEDGEMENT}

The authors wish to acknowledge the University Teknologi PETRONAS (UTP) for funding this study through the YUTP Research Project Grant (015LC0-003); Malaysia Ministry of Environment (Jabatan Alam Sekitar) for providing oil spill location data; ESA Copernicus and ASF Alaska for the free SAR imagery.

\section{REFERENCES}

Balogun, A. L., Yekeen, S. T., Pradhan, B. \& Althuwaynee, O. F. 2020. Spatio-Temporal Analysis Of Oil Spill Impact And Recovery Pattern Of Coastal Vegetation And Wetland Using Multispectral Satellite Landsat 8-Oli Imagery And Machine Learning Models. 12, 1225.

Chen, J., Zhang, W., Wan, Z., Li, S., Huang, T. \& Fei, Y. 2019. Oil Spills From Global Tankers: Status Review And Future Governance. Journal Of Cleaner Production, 227, 20-32.

Fingas, M. 2016. Oil Spill Science And Technology, Gulf Professional Publishing.

Fingas, M. \& Brown, C. 2018. A Review Of Oil Spill Remote Sensing. Sensors, 18, 91.

Fingas, M. \& Brown, C. J. M. P. B. 2014. Review Of Oil Spill Remote Sensing. 83, 9-23.

Gallego, A.-J., Gil, P., Pertusa, A. \& Fisher, R. B. 2019. Semantic Segmentation Of Slar Imagery With Convolutional Lstm Selectional Autoencoders. $11,1402$.

Gallego, A. J., Gil, P., Pertusa, A. \& Fisher, R. B. 2018. Segmentation Of Oil Spills On Side-Looking Airborne Radar Imagery With Autoencoders. Sensors (Basel), 18.

Jiao, Z., Jia, G. \& Cai, Y. 2019. A New Approach To Oil Spill Detection That Combines Deep Learning With Unmanned Aerial Vehicles. Computers \& Industrial Engineering, 135, 1300-1311.

Krestenitis, M., Orfanidis, G., Ioannidis, K., Avgerinakis, K., Vrochidis, S. \& Kompatsiaris, I. 2019. Oil Spill Identification From Satellite Images Using Deep Neural Networks. 11, 1762.

Leifer, I., Lehr, W. J., Simecek-Beatty, D., Bradley, E., Clark, R., Dennison, P., Hu, Y., Matheson, S., Jones, C. E., Holt, B., Reif, M., Roberts, D. A., Svejkovsky, J., Swayze, G. \& Wozencraft, J. 2012. State Of The Art Satellite And Airborne Marine Oil Spill Remote Sensing: Application To The Bp Deepwater Horizon Oil Spill. Remote Sensing Of Environment, 124, 185-209.

Liu, P., Zhao, C., Li, X., He, M. \& Pichel, W. 2010. Identification Of Ocean Oil Spills In Sar Imagery Based On Fuzzy Logic Algorithm. International Journal Of Remote Sensing, 31, 4819-4833.

Ma, L. 2016. Support Tucker Machines Based Marine Oil Spill Detection Using Sar Images.

Mera, D., Fernández-Delgado, M., Cotos, J. M., Viqueira, J. R. R. \& Barro, S. 2017. Comparison Of A Massive And Diverse Collection Of Ensembles And Other Classifiers For Oil Spill Detection In
Sar Satellite Images. Neural Computing And Applications, 28, 1101-1117.

Michel, J. \& Fingas, M. 2015. Oil Spills: Causes, Consequences, Prevention, And Countermeasures. Fossil Fuels, Columbia: Research Planning, Inc.

Migliaccio, M., Nunziata, F. \& Gambardella, A. Polarimetric Signature For Oil Spill Observation. 2008 Ieee/Oes Us/Eu-Baltic International Symposium, 2008. Ieee, 1-5.

Ozigis, M. S., Kaduk, J. D. \& Jarvis, C. H. 2019a. Mapping Terrestrial Oil Spill Impact Using Machine Learning Random Forest And Landsat 8 Oli Imagery: A Case Site Within The Niger Delta Region Of Nigeria. Environmental Science And Pollution Research, 26, 3621-3635.

Ozigis, M. S., Kaduk, J. D., Jarvis, C. H., Da Conceição Bispo, P. \& Balzter, H. 2019b. Detection Of Oil Pollution Impacts On Vegetation Using Multifrequency Sar, Multispectral Images With Fuzzy Forest And Random Forest Methods. Environmental Pollution, 113360.

Robbe, N. \& Hengstermann, T. 2006. Remote Sensing Of Marine Oil Spills From Airborne Platforms Using Multi-Sensor Systems. Water Pollution Viii: Modelling, Monitoring Management 1, 347-355.

Singha, S., Bellerby, T. J. \& Trieschmann, O. Detection And Classification Of Oil Spill And Look-Alike Spots From Sar Imagery Using An Artificial Neural Network. 2012 Ieee International Geoscience And Remote Sensing Symposium, 22-27 July 2012 2012. 5630-5633.

Singha, S., Bellerby, T. J. \& Trieschmann, O. 2013. Satellite Oil Spill Detection Using Artificial Neural Networks. Ieee Journal Of Selected Topics In Applied Earth Observations And Remote Sensing, 6, 2355-2363.

Skøelv, Å. \& Wahl, T. J. T. R., Norwegian Defence Research Establishment 1993. Oil Spill Detection Using Satellite Based Sar, Phase 1b Competition Report.

Topouzelis, K., Karathanassi, V., Pavlakis, P. \& Rokos, D. 2007. Detection And Discrimination Between Oil Spills And Look-Alike Phenomena Through Neural Networks. Isprs Journal Of Photogrammetry And Remote Sensing, 62, 264270.

Vyas, G., Bhan, A. \& Gupta, D. 2015. Detection Of Oil Spills Using Feature Extraction And Threshold Based Segmentation Techniques. 2015 2nd International Conference On Signal Processing And Integrated Networks (Spin).

Wan, J. \& Cheng, Y. Remote Sensing Monitoring Of Gulf Of Mexico Oil Spill Using Envisat Asar Images. 2013 21st International Conference On Geoinformatics, 20-22 June 20132013. 1-5.

Yekeen, S., Balogun, A. \& Aina, Y. 2019. Early Warning Systems And Geospatial Tools: Managing Disasters For Urban Sustainability. In: Leal Filho, W., Azul, A. M., Brandli, L., Özuyar, P. G. \& Wall, T. (Eds.) Sustainable Cities And 
Communities. Cham: Springer International Publishing.

Yu, F., Xue, S., Zhao, Y. \& Chen, G. 2018. Risk Assessment Of Oil Spills In The Chinese Bohai Sea For Prevention And Readiness. Marine Pollution Bulletin, 135, 915-922.

Zeng, K. \& Wang, Y. 2020. A Deep Convolutional Neural Network For Oil Spill Detection From Spaceborne Sar Images. 12, 1015.

Zhang, J., Feng, H., Luo, Q., Li, Y., Wei, J. \& Li, J. 2020. Oil Spill Detection In Quad-Polarimetric Sar
Images Using An Advanced Convolutional Neural Network Based On Superpixel Model. 12, 944.

Zhao, S., Zhang, D. M. \& Huang, H. W. 2020. Deep Learning-Based Image Instance Segmentation For Moisture Marks Of Shield Tunnel Lining. Tunnelling And Underground Space Technology, 95, 103156. 